\title{
Article \\ Evaluation of IL1 $\beta$ and IL6 Gingival Crevicular Fluid Levels during the Early Phase of Orthodontic Tooth Movement in Adolescents and Young Adults
}

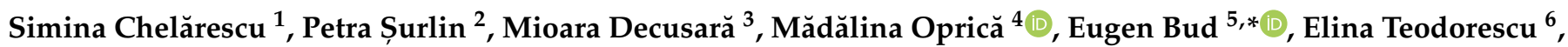 \\ Mahmoud Nabil Elsaafin ${ }^{5}$ and Mariana Păcurar ${ }^{5}$
}

1 Faculty of Dentistry, George Emil Palade University of Medicine and Pharmacy, 540139 Targu Mures, Romania; chelarescu.simina@yahoo.com

2 Department of Periodontology, Faculty of Dentistry, University of Medicine and Pharmacy of Craiova, 200349 Craiova, Romania; petra.surlin@umfcv.ro

3 Department of Orthodontics, Faculty of Dentistry, Dunarea de Jos University of Medicine and Pharmacy of Galati, 800008 Galati, Romania; mioara.decusara@ugal.ro

4 Center of Advanced Medical and Pharmaceutical Research/CCAMF, George Emil Palade University of Medicine and Pharmacy, 540139 Targu Mures, Romania; madalina.nedelcu@umfst.ro

5 Department of Orthodontics, Faculty of Dentistry, George Emil Palade University of Medicine and Pharmacy Targu Mures, 38 Gheorghe Marinescu Street, 540139 Targu Mures, Romania; nabil.elsaafinmahmoud@umfst.ro (M.N.E.); Mariana.pacurar@umfst.ro (M.P.)

6 Department of Orthodontics, Faculty of Dentistry, Carol Davila University of Medicine and Pharmacy, 050474 Bucuresti, Romania; elina.teodorescu@umfcd.ro

* Correspondence: eugen.bud@umfst.ro; Tel.: +40-744437661

Citation: Chelărescu, S.; Șurlin, P.; Decusară, M.; Oprică, M.; Bud, E.; Teodorescu, E.; Elsaafin, M.N.; Păcurar, M. Evaluation of IL1 $\beta$ and IL6 Gingival Crevicular Fluid Levels during the Early Phase of Orthodontic Tooth Movement in Adolescents and Young Adults.

Appl. Sci. 2021, 11, 521.

https://doi.org/10.3390/ app11020521

Received: 11 December 2020

Accepted: 2 January 2021

Published: 7 January 2021

Publisher's Note: MDPI stays neutral with regard to jurisdictional clai$\mathrm{ms}$ in published maps and institutional affiliations.

Copyright: (C) 2021 by the authors. Licensee MDPI, Basel, Switzerland. This article is an open access article distributed under the terms and conditions of the Creative Commons Attribution (CC BY) license (https:// creativecommons.org/licenses/by/ $4.0 /)$.
Abstract: Background: The crevicular fluid analysis represents a useful diagnosis tool, with the help of which noninvasive cellular metabolic activity can be analyzed. The aim of the study is to investigate comparatively IL1 $\beta$ and IL6 in the gingival crevicular fluid of clinically healthy adolescents and young adults during the acute phase of orthodontic treatment. Methods: Gingival crevicular fluid was collected from 20 patients (aged between 11 and 28) undergoing orthodontic treatment. Measurements were taken before (T0) and after $24 \mathrm{~h}$ after distalization forces were activated (T1). IL1 $\beta$ and IL 6 were analyzed using Elisa tests. The statistical tests used were twosided $t$ tests. Results: Between the two time periods there was a significant raise both in the crevicular fluid rate $(0.57 \mu \mathrm{L}$ at T0 vs. $0.95 \mu \mathrm{L}$ at T1, $p=0.001)$ and in IL1 $\beta$ levels $(15.67 \mathrm{pg} / \mu \mathrm{L}$ at T0 vs. $27.94 \mathrm{pg} / \mu \mathrm{L}$ at T1, $p=0.009)$. We were able to identify IL6 only in a third of the sites. There is a significantly increased level of IL $\beta$ at T1 in adolescents, more than in young adults $(42.96 \mathrm{pg} / \mu \mathrm{L}$ vs. $17.93 \mathrm{pg} / \mu \mathrm{L}, p=0.006$ ). Conclusions: In the early stage of orthodontic treatment, the periodontal tissues of adolescents are more responsive to orthodontic forces than those of young adults.

Keywords: cytokines; dentistry; ELISA test; gingival crevicular fluid; orthodontic treatment; orthodontics

\section{Introduction}

The crevicular fluid analysis represents a useful diagnosis tool, which can be of great interest both in the periodontal disease and orthodontic therapy, with the help of which the noninvasive cellular metabolic activity can be analyzed [1].

The crevicular/sulcular fluid is found at the level of gingival sulcus, originating in the vascular plexus in the corion. Its nature is a controversial topic in literature. The gingival fluid is considered to be a transudate, which becomes an exudate in the presence of inflammation 272]. The presence of gingival fluid was initially studied by Jens Waerhaug in 1952 [1], through the experimental administration of ink particles in the crevicular sulci of dogs. One hour after the administration, a raise in volume was observed. $48 \mathrm{~h}$ later, almost a complete removal of these particles was noticed, thus suggesting a possible 
mechanical role in removing foreign particles from the gingival crevicular fluid. This role was confirmed by administrating pathogenic bacterial complexes in germ free gingival sulci. After the initial epithelial necrosis, clinical signs of inflammation were detected, with the induction of an inflammatory exudate. However, after $48 \mathrm{~h}$, the gingival sulcus returned to the normal aspect [1].

In addition to the volumetric raises caused by the presence of inflammation, increases in the flow rate of gingival fluid were observed in other pathological or physiological conditions, such as: the circadian rhythm (in the morning, between 6-10 a.m an increase in flow rate was observed), physiological factors (sexual hormones, mastication), smoking, periodontal surgery (a certain increase in the volume appeared in the post-surgery phase) [2]. In case of a minor inflammation, the gingival fluid rate is $0.05-0.2 \mu \mathrm{L} / \mathrm{min}$. Its daily flow rate is between $0.5-2.4 \mathrm{~mL}$ [3].

The gingival fluid is composed of substances derived from serum, host inflammatory cells, periodontal cells and oral bacteria. The molecular components include electrolytes, proteins, cytokines, antibodies, antigens, bacterial or local enzymes [4].

A detailed analysis of these components provides us with useful information regarding the cellular metabolic activity during either the periodontal therapy or the orthodontic treatment. Certain components of the gingival fluid being quantified, they can act as biomarkers in identifying periodontal diseases, monitoring and evaluating the response to the periodontal treatment $[5,6]$.

Evaluating the biomarkers during the orthodontic therapy allows us to monitor the effectiveness of the treatment, to anticipate the future outcome and also to individualize the treatment according to the response of tissue to different force intensities [3,4]. This is significant especially in adult patients, where the pace of the periodontal restructuring occurs in a longer time than in juvenile patients [7]. Through physiological dental migrations, the periodontal tissues are continuously subjected to remodeling action. While the physiologic migrations are achieved through a slow process, the orthodontic migrations can occur through both accelerated and slow mechanisms. These movements are influenced by the intensity of the applied forces, or by the biological response of the periodontal ligament. Inflammatory reactions that appear in response to mechanical stimuli determine periodontal inflammation, thus determining bone remodeling. The bone resorption necessary for orthodontic movement is achieved by applying low intensity mechanical forces in the pressure side of the periodontium, thus the cellular and tissular physiology remain intact. Increased mechanical forces affect the periodontal tissue determining cell necrosis, the appearance of a hyaline tissue and an acellular area between the periodontal ligament and the adjacent bone, thus slowing down the orthodontic movement [8].

Orthodontic forces applied onto the tooth cause increased tissue pressure, leading to accelerated bone remodeling. Thus, alterations appear at extracellular matrix, with alterations of the cell shape and cytoskeletal conformation. As a result of these changes, there are variations in the gingival fluid flow rate and composition. Nevertheless, the orthodontic induced inflammation and inflammatory markers are transient. The most significant changes appear during the initial acute phase. The initial phase occurs $24-48$ $\mathrm{h}$ after the orthodontic forces have been applied. This phase is characterized by a rapid and short movement of the tooth. Due to the compression of the periodontal ligament on the one hand, and the stretching on the other hand, this phase is characterized by an acute inflammatory response, represented by vasodilation, chemo-attraction of the inflammatory cells, osteoblast and osteoclast progenitor cells [7].

The orthodontically induced inflammation is accompanied by an increase of the inflammatory mediators (cytokines, prostaglandins, leukotrienes), enzymes (metalloproteinase, alkaline phosphatase, aspartate aminotransferase), growth factors, neuropeptides, suggesting the fact that there are many factors involved in bone remodeling (immune, endocrines, nervous). Cytokines are the first factors that influence the stimulation or inhibition of the biomechanical reactions that appear during the orthodontically induced 
inflammation. The cytokines involved in the bone remodeling process are interleukin 1 $(\alpha, \beta)$, interleukin 6 and tumor necrosis factor (TNF $\alpha)$ [9].

The objective of the study is the comparative investigation of inflammatory mediators such as the cytokines in clinically healthy adolescents and young adults during the acute phase of orthodontic treatment.

Therefore, the null hypothesis (H0) underlying this study is that there is no statistically significant difference between the inflammatory mediators (such as the cytokines) between groups of healthy adolescents and young adults, respectively, during the acute phase of orthodontic treatment.

\section{Materials and Methods}

\subsection{Study Design}

The study consisted of 20 patients ( 11 females, 9 males) aged between 11-16 (8 patients) and 17-28 (12 patients), pertaining to the Orthodontics and Dentofacial Orthopedics Clinic of the Medical Dental School George Emil Palade University of Medicine and Pharmacy from Targu Mures. The study was conducted in accordance with the Declaration of Helsinki and was approved by the Ethics Committee of George Emil Palade University of Medicine and Pharmacy (897/08 May 2020). A written informed consent was obtain from all patients, prior to the investigations.

\subsection{Inclusion Criteria}

- Indication for orthodontic treatment, with premolar extraction (all malocclusion classes were considered)

- No systemic affections

- Periodontal health

Periodontal health was assessed using the basic periodontal examination (BPE), using a World Health Organization probe (WHO probe 550B, LM-Instruments, Prainen, Finland). The study included only patients with scoring codes 0 (no bleeding on probing, probing pocket depths $<3.5 \mathrm{~mm}$, no calculus or overhanging restorations). Moreover, no evident radiographic bone loss was present [10].

\subsection{Exclusion Criteria}

- Systemic diseases s (systemic diseases that have a major role in the loss of periodontal tissues were excluded, such as: genetic, metabolic and endocrine diseases, inflammatory diseases, acquired immunodeficiency diseases

- Periodontal diseases (BPE scoring $>0$, radiographic bone loss)

- Systemic treatment with antibiotics or anti-inflammatory treatment in the month preceding the study.

\subsection{Orthodontic Treatment}

The orthodontic treatment consisted of the application through bonding of metallic brackets (3M, Unitek, CA, USA) with a 0.22 inch slot and orthodontic tubes (3M, Monrovia, CA, USA), using the MBT technique on the maxillary and mandibular arches of young patients with different dento-maxillary anomalies. Brackets were applied through self-etching technique using Transbond XT material (3M, Unitek, CA, USA), due to its hybrid filling and high resistance to compression and bending [11]. Medium forces were applied, using closed coil springs and $0.19 \times 0.25$ SS wires (Forestadent, Pforzheim, Germany). All patients were clinically (basic periodontal examination) and radiologically evaluated, monitoring the quality of periodontal tissues in order to detect incipient signs of periodontal diseases.

\subsection{Collection of the Crevicular Fluid}

The crevicular fluid was collected from the distal zone of the canine, before the activation of the orthodontic treatment (control group) and $24 \mathrm{~h}$ after its activation (study 
group). In order to avoid a potential contamination with blood from the test strips through multiple insertions in the gingival sulcus, we defined the right superior canine (tooth number 1.3) as situs for collection of IL1 $\beta$ and the left superior canine (tooth number 2.3) for collection of IL6.

Before the collection, patients lightly rinsed their mouth with water; afterwards, the harvest zone was gently dried with the air from triple spray. The teeth were isolated using a cotton roll, and then using another roll, the supragingival bacterial plaque was gently removed. The harvest of gingival fluid was done by using filter paper strips (Periopaper ProFlow Inc, Amityville, NY, USA) (Figure 1), which were introduced in the gingival sulcus at a depth of $1 \mathrm{~mm}$ and maintained for $30 \mathrm{~s} \mathrm{(Figure} \mathrm{2).} \mathrm{The} \mathrm{quantity} \mathrm{of} \mathrm{crevicular} \mathrm{fluid}$ volume was measured using a Periotron 8000 device (Pro-Flow Inc., Amityville, New York, NY, USA) which measures the capacitance modification on the filter paper (Figure 3). The filter paper was introduced between the two plates of the device; following that, the device transmitted the modification to a digital screen. The scores are then converted to microliters using mlconvert.exe software (Ora Flow, Amityville, NY, USA). After each measuring, Periotron's electrodes were cleaned using a sterile compress lightly damped in saline to eliminate the risk of contamination.

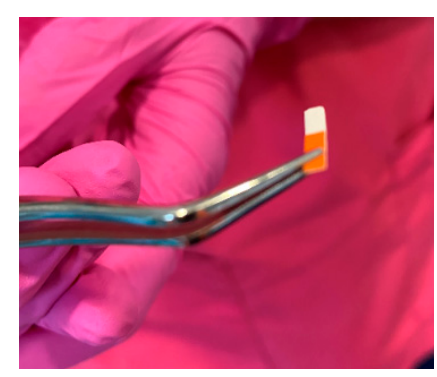

Figure 1. Periopaper strip.

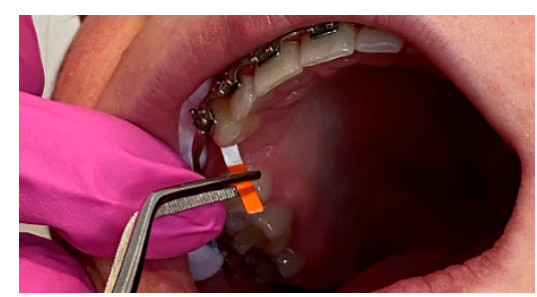

Figure 2. Sample harvesting.

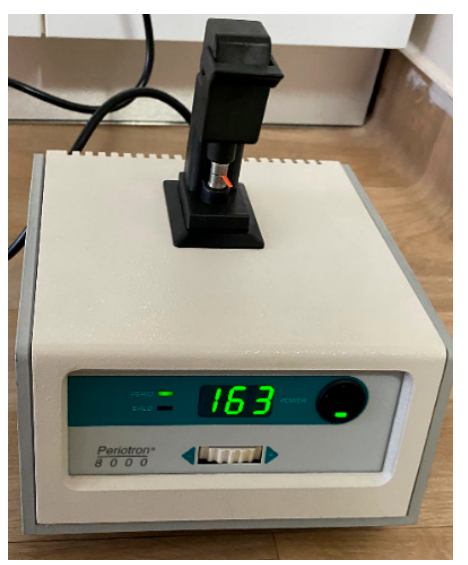

Figure 3. Measuring gingival fluid volume using Periotron.

The strips contaminated with blood were not used but discarded. After reading the strips, they were introduced in a Eppendorf safe-lock crio-tube, which contained 
250 microliters $(\mu \mathrm{L})$ of phosphate buffer solution (Figure 4). The crio-tubes were than deposited in waxed boxes (Figure 5), and, shock-cooled to $-80{ }^{\circ} \mathrm{C}$ until the harvesting and lab processing was finalized.

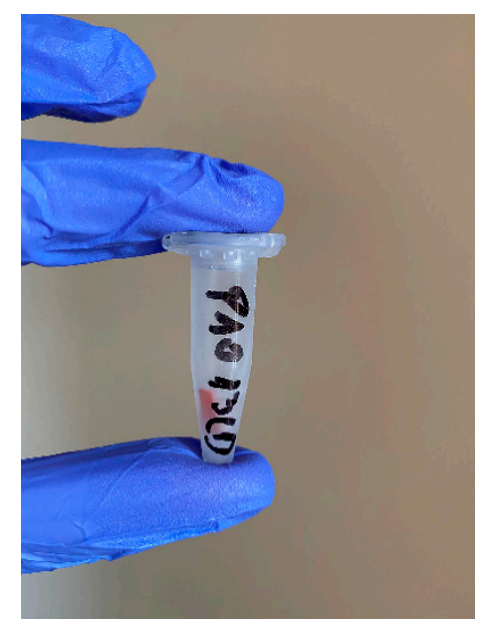

Figure 4. Depositing the sample in Eppendorf tube.

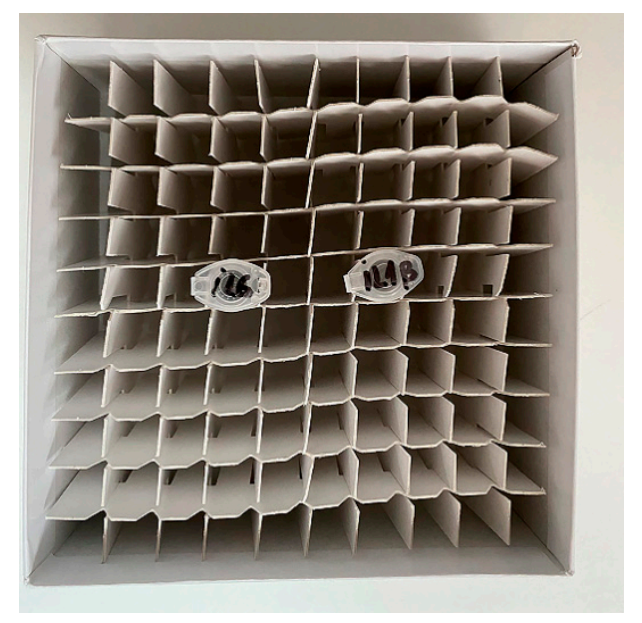

Figure 5. Depositing the tubes in waxed boxes that are stacked in the freezer.

The devices, equipment, and materials utilized were: a Dynex Dsx automated ELISA system Analyzer (Dynex Technologies, Chantilly, VA, USA), a Vortex, a MiniSpin centrifuge (Eppendorf, Hamburg, Germany), automatic pipettes (20-200 $\mu \mathrm{L}, 100-1000 \mu \mathrm{L}-$ Scilogex, Rocky Hill, CT, USA), single-use tips for pipettes (Eppendorf, Hamburg, Germany), distilled water (Chimopar, Bucharest, Romania), Periopaper strips (Oraflow, New York, NY, USA), single-use (disposable) gloves and a consultation kit.

\subsection{Immuno-Enzymatic Analysis of IL1 $\beta$ and IL6}

The immuno-enzymatic analysis of IL1 $\beta$ and IL6 was done in the CCAMF Center (Medical and Pharmaceutical Advance Research Center), from George Emil Palade University of Medicine and Pharmacy of Targu Mures, Targu Mures, Romania, strictly following the protocol as indicated by the kit manufacturer, both for IL1 $\beta$ and IL6 (i.e., different protocols are required by the manufacturer for each of the two cytokines).

The reagents are brought to room temperature and prepared according to the work protocol. The samples are defrosted, vortexed and centrifuged.

The test uses ELISA sandwich-type quantitative immune-enzymatic method (R\&D Systems Co., Minneapolis, MN, USA). 
The first stage of the test consists of incubation of the standards and samples added to the papered well with a monoclonal antibody specific for IL- $1 \beta$. The IL- $1 \beta$ present in the sample will link to the antibody fixed in the well, forming an antibody-antigenic complex. After the first incubation stage, there follows a cleansing step using a washing buffer solution that removes the excess antigens that did not link to the antibodies in the wells.

The second stage consists of adding the conjugate (polyclonal antibody specific for IL-1 $\beta$ marked with an enzyme- peroxidases from radish root) followed by an incubation period that will determine the creation of an antibody-antigen-antibody complex (sandwich principle). The incubation is followed by another cleaning process to remove the unlinked polyclonal antibody.

Next, the sublayer is added (a solution consisting of a mixture of peroxide hydrogen and a composite cromogen-tetrametilbenzydine) that will produce a coloring reaction, depending on the quantity of linked IL-1 $\beta$ in the sample. After adding a stopping solution with sulfuric acid, the color ranges from blue to yellow. The absorbance of each well will be read spectrophotometrically by the analyzer; the software will determine a standard curve by representing the absorbance relative to the concentration for each standard. The IL-1 $\beta$ concentration will be determined by reading the absorbance degree for each well and calibrating it using a calibration curve, with the cytokine concentration being directly proportional to the intensity of the well color.

The protocol schema for IL1 $\beta$ and IL6 is shown below (Figures 6 and 7).

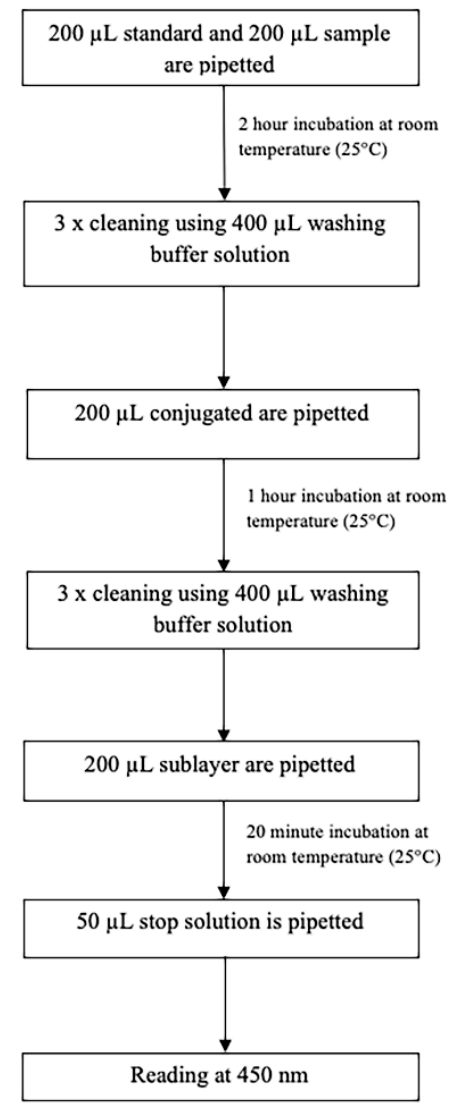

Figure 6. Protocol for IL1 $\beta$. 


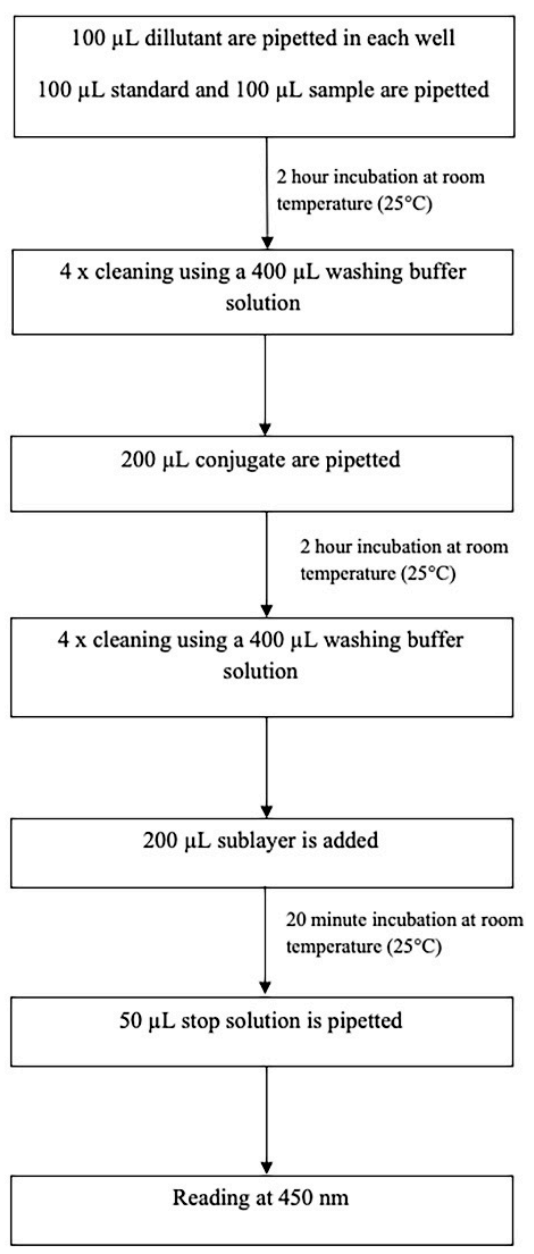

Figure 7. Protocol for IL6.

The utilized kits recognize the human natural and recombined $6 / 1 \beta$ interleukin.

No interference or significant cross reactivity with other human recombined interleukins were observed.

The concentration of interleukin in the gingival sulcus were calculated using the following formula: total cytokine quantity (pictograms $/$ microliters $-\mathrm{pg} / \mu \mathrm{L}$ ) $=$ identified cytokine quantity $(\mathrm{pg} / \mathrm{mL}) \times$ dilution volume $(250 \mu \mathrm{L}) /$ crevicular fluid volume $(\mu \mathrm{L})$.

\subsection{Statistical Method}

The statistical software used was IBM SPSS Statistics Base version 26 (International Business Machines Corporation, Armonk, NY, USA), and we deployed both descriptive statistics to understand the distribution of data, and two-sample t-tests to calculate statistical significance levels in the different groups. Statistical significance was considered at $95 \%$ confidence level in all analyses, corresponding to $p$ values of $<0.05$. We have performed an a priori power analysis using IBM SPSS native power analysis tool to better ground the relevance of our study. We utilized a standard alpha measure of 0.05 , the generally utilized level of alpha in medical studies. The power analysis was tailored to our two-sided test chosen for our methodology.

Due to the lack of prevalence data and reliable statistical information in our region, we performed a literature review in order to identify the most similar study whose data and results were found appropriate to be used as an estimation of the population means and standard deviations in our power analysis—-the study chosen was performed by Vujacic A et al. [12].

Thus, we obtained a power level of 0.69 , close to the commonly sought threshold of 0.8 . Despite falling slightly below this threshold, we consider the a priori analysis acceptable, 
due to two factors. Firstly, due to the lack of statistical information and prevalence in the population, we had to rely on a literature review to estimate power a priori, a methodology that can be more sensitive to results. Secondly, we performed an a posteriori power analysis based on our actual results, showing a strong power factor of 0.89 that can be the grounds for future articles developing studies on this subject.

\section{Results}

The patients maintained a good oral hygiene. None of the patients suffered significant changes in periodontal indices during the two time periods. No bleeding on probing (BP) or probing pocket depth (PPD) were detected $24 \mathrm{~h}$ before or after the initiation of the orthodontic treatment.

GCF analysis revealed detectable values for IL1 $\beta$ at both time periods. We were able to identify IL6 only on a third of the sites, the other values being below the test detection threshold. Thus, the mean value identified for IL6 at T0 is $0.14 \mathrm{pg} / \mu \mathrm{L}$, and for T1 $1.27 \mathrm{pg} / \mu \mathrm{L}$, values that were not taken into consideration in the statistics. Moreover, the identified values for IL6 were lower than those identified for IL1 $\beta$.

The inflammation caused by the orthodontic movement is followed by an increase in both gingival crevicular levels and inflammatory mediators. Table 1 presents the values $24 \mathrm{~h}$ before (T0) and after (T1) the initiation of the orthodontic treatment for gingival crevicular volume and for IL1 $\beta$ levels. Both of these parameters showed increased values at T1. Between the two time periods, there were significant changes both in the crevicular fluid rate $(p=0.001)$ and in IL1 $\beta$ levels $(p=0.009)$.

Table 1. Gingival crevicular volumes ( $\mu \mathrm{L})$, IL1 $\beta$ values $(\mathrm{pg} / \mu \mathrm{L})$ before and after $24 \mathrm{~h}$ after the initiation of orthodontic treatment.

\begin{tabular}{ccccc}
\hline & $\begin{array}{c}\text { Gingival } \\
\text { Crevicular Volume } \\
\text { T0 }(\mu \mathrm{L})\end{array}$ & $\begin{array}{c}\text { Gingival } \\
\text { Crevicular Volume } \\
\text { T1 }(\mu \mathrm{L})\end{array}$ & $\begin{array}{c}\text { IL1 } \beta-T 0 \\
(\mathbf{p g} / \mu \mathbf{L})\end{array}$ & $\begin{array}{c}\text { IL1 } \beta-T 1 \\
(\mathbf{p g} / \mu \mathrm{L})\end{array}$ \\
\hline Mean & 0.57 & 0.95 & 15.67 & 27.94 \\
SE & 0.05 & 0.10 & 2.21 & 3.92 \\
SD & 0.23 & 0.46 & 9.89 & 17.54 \\
Minimum & 0.28 & 0.35 & 6.42 & 13.09 \\
Maximum & 0.93 & 1.54 & 37.64 & 69.91 \\
Confidence level (95\%) & 0.11 & 0.21 & 4.62 & 8.21 \\
\hline$p$ value & & 0.001 & \multicolumn{3}{c}{0.009} \\
\hline
\end{tabular}

Tables 2 and 3 show a comparison of IL1 $\beta$ values by age groups in the two time periods. Our results indicate higher levels of IL1 $\beta$ in adolescents than in young adults before and after the initiation of the orthodontic treatment. In adolescents, the mean value for IL1 $\beta$ raised from $22.77 \mathrm{pg} / \mu \mathrm{L}$ at $\mathrm{T} 0$ to $42.96 \mathrm{pg} / \mu \mathrm{L}$ at T1, while in the age group represented by young adults, the concentration of IL1 $\beta$ raised from $11.28 \mathrm{pg} / \mu \mathrm{L}$ at T0 to $17.93 \mathrm{pg} / \mu \mathrm{L}$ at T0. Adolescents had a statistically significant higher level of IL1 $\beta 24 \mathrm{~h}$ before $(p=0.031)$ and after the initiation of orthodontic treatment $(p=0.006)$.

Table 2. IL1 $\beta$ values $(\mathrm{pg} / \mu \mathrm{L})$ in adolescents (11-16 years old) and young adults (17-28 years old) at T0.

\begin{tabular}{ccc}
\hline Age & $\mathbf{1 7 - 2 8}$ Years & $\mathbf{1 1 - 1 6}$ Years \\
\hline Mean & 11.28 & 22.77 \\
SE & 1.01 & 4.30 \\
SD & 3.66 & 12.17 \\
Minimum & 6.42 & 6.47 \\
Maximum & 17.58 & 37.64 \\
Confidence level (95\%) & 2.21 & 10.17 \\
\hline$p$ value & & \\
\hline
\end{tabular}


Table 3. IL1 $\beta$ values $(\mathrm{pg} / \mu \mathrm{L})$ in adolescents (11-16 years old) and young adults (17-28 years old) at T1.

\begin{tabular}{ccc}
\hline Age & $\mathbf{1 7 - 2 8}$ Years & $\mathbf{1 1 - 1 6}$ Years \\
\hline Mean & 17.93 & 42.96 \\
SE & 1.53 & 6.71 \\
SD & 5.311 & 18.99 \\
Minimum & 13.09 & 20.28 \\
Maximum & 27.62 & 20.28 \\
Confidence level (95\%) & 3.37 & 15.88 \\
\hline$p$ value & & \\
\hline
\end{tabular}

\section{Discussion}

Orthodontic teeth movements determine an inflammatory aseptic transitory response. In the first stages of the orthodontic movements, there is an increase in the vascular permeability, accompanied by a leucocyte infiltrate. Immunity cells, together with native cells (fibroblasts, osteoblasts), are responsible for the production of inflammatory cytokines. At the level of the crevicular fluid of the teeth involved in the orthodontic movement, high concentrations of inflammatory cytokines like IL1, IL2, IL6, IL8, TNF $\alpha$, IFN $\gamma$ were identified [13]. Out of the wide range of inflammatory mediators we chose IL $1 \beta$ and IL6 pro-inflammatory cytokines, due to their role in the destructive process of the marginal periodontium.

IL1 $\beta$ and IL6 are among the first cytokines that modified the concentration of the crevicular fluid at the moment of application of orthodontic forces. Both cytokines are mediators of the inflammation, associated with inflammation determined by orthodontic movements. IL1 $\beta$ is a key mediator, being involved both in the inflammation determined by orthodontic movements, and in various immune reactions. IL6 is a mediator of the bone remodeling, being in direct connection with the bone cells [12,14-16].

Multiple roles are attributed to IL6: part of the final differentiation of B lymphocytes in plasmocytes, influence in proliferation of T cells, stimulus of the DNA synthesis, intervening factor in the bone remodeling process through the inhibition of collagen synthesis. There is a correlation between the tissular level of IL6 and the inflammation present in the tissues. Through a synergetic action with IL1 $\beta$, it stimulates bone resorption by the osteoclasts $[17,18]$.

In this article we studied the concentration of interleukin $1 \beta$ and IL6 at the beginning of the orthodontic treatment and $24 \mathrm{~h}$ after its activation, at the level of distal gingival sulcus of the canine on which distalization forces are applied. In the literature, we identified similar studies, which quantify the pro-inflammatory cytokines associated with the orthodontic treatment at different times after the treatment started ( $24 \mathrm{~h}, 72 \mathrm{~h}, 3$ months). In those studies, we found different results reporting on: the total cytokine quantity in each sample (picograms), the weight reported to the cytokine mass, the cytokine quantity reported to the strip exposure time (picograms/60 s), or the cytokine concentration (the value of cytokine in each sample studies relative to the volume- $\mathrm{pg} / \mathrm{mL}$ or $\mathrm{pg} / \mu \mathrm{L}$ ).For this study, we chose the latter method for our reporting. The total cytokine quantity (pg/situs) is equivalent to the identified cytokine quantity, multiplied by the dilution volume $(250 \mu \mathrm{L})$. The interleukin concentration was calculated as being the total cytokine quantity, relative to the volume of crevicular fluid in the situs (pg/ $\mu \mathrm{L})$ [19]. As opposed to other cited studies, our study did not identify interleukin 6 for more than a third of the patients, with our results being under the level needed for detection. The fact that only $1 / 3$ of the tests obtained IL6 levels above the test detection level, stresses our results, showing that $24 \mathrm{~h}$ after the activation of the orthodontic forces, some amount of inflammation is present, but generally not in high levels. In the literature, similar quantities collected from the gingival sulcus were identified by using high sensitivity tests.

In the study led by Emel Sari and Cihan Ucar [20], the authors compared the concentration of IL $1 \beta$ for patients undergoing orthodontic treatment with mini-implants, comparing the concentration of cytokine in the mini-implant sulcus, and at the level of 
the distalized canine, respectively. Due to the extended methodological similarities with our study, the results obtained by Emel Sari and Cihan Ucar [20] in their work are highly comparable. Firstly, the authors opted for an identical reporting of results, identifying the interleukin concentration relative to the gingival fluid volume $(\mathrm{pg} / \mu \mathrm{L})$. Furthermore, the dilution volume used $(250 \mu \mathrm{L})$ is also identical. The authors obtained similar results both at the $24 \mathrm{~h}$ threshold at the level of distalized canines $(37.8 \pm 6.7 \mathrm{pg} / \mu \mathrm{L})$, and at the control situs level $18.3 \pm 3.4 \mathrm{pg} / \mu \mathrm{L}$.

With regard to the collected crevicular fluid volume, our results are similar to others obtained in the literature. For collecting the crevicular fluid we used the paper points which possess a narrow absorption limit emphasizing the importance of avoiding bleeding during sampling [21]. In our study, the average crevicular volume $24 \mathrm{~h}$ after activating the orthodontic apparatus was $0.955 \mu \mathrm{L}$. The average value obtained before activation was $0.57 \mu \mathrm{L}$. The results are similar to the ones obtained by S. Drummond and co. They identified a crevicular fluid volume $24 \mathrm{~h}$ after activation of $0.54 \pm 19 \mu \mathrm{L}$, while the volume collected level at the control situs was $0.66 \pm 21 \mu \mathrm{L}$ [22].

The metabolic activity of the periodontal tissues is also influenced by their physiological characteristics. Thus, an important factor that needs to be considered regarding the periodontal tissue remodeling during the orthodontic therapy is the patient's age [23]. The alveolar bone density grows with age and will thus generate a different biological response in teen/adult patients relative to children. A study conducted by Grzibovskis shows that the reduced level of pro-inflammatory cytokines in adult patients is due to the decreased metabolic activity of the periodontal ligament for this age group (2011). Therefore, the periodontal tissue remodeling and the release of inflammatory molecules decrease with growth in age. In our study, we observed an increased IL $1 \beta$ concentration for young patients $(42.96 \mathrm{pg} / \mu \mathrm{L})$ compared to adult patients $(17.93 \mathrm{pg} / \mu \mathrm{L}))$ in the first $24 \mathrm{~h}$ after the activation of orthodontic therapy. Similar results were also obtained by Amila Vujacic et al. [12]. The authors compared the concentration of IL $1 \beta$ and IL6 in the crevicular fluid for adults and children during the orthodontic treatment, at different time intervals. Their results were reported in a different manner from ours (mass of interleukin per $60 \mathrm{~s}$ ). They identified a similar concentration of IL1 $\beta$ at the control teeth for both adults and children, with higher concentrations identified at the IL $1 \beta$ level. In regards to the teeth affected by orthodontic forces, IL1 $\beta$ had higher values for both adults and children, at 24 and $168 \mathrm{~h}$ after time of application of the device. Overall, the concentration of IL1 $\beta$ was higher for children compared to adults, with statistically significant differences at the initial time of the device activation and $168 \mathrm{~h}$ later $[24,25]$.

Our research is in line with those identified in the literature, suggesting that initial tooth movements occur more rapidly in adolescents than in young adults. However, many variables could alter the results of the present preliminary report. In fact the lingual or vestibular bracket positioning has been demonstrated to have an effect on periodontal parameters [26]. Additionally, treatment time is also a significant variable [27]. Therefore, further clinical research is needed on the topic.

The main limitation of this study is represented by the time periods of the measurement of the gingival crevicular fluid levels, with the study having been conducted only at T0 and T1. Our initial plan was to analyze the crevicular fluid levels at multiple time periods (i.e., in addition to the current analyzed timing, we planned to also analyze results at T2 and T3, respectively at 1 month and 3 months after the activation of the orthodontic forces), however our study plan was amended due to the COVID-19 19 crisis and we were required to limit our research. The second limitation of the study is the sample size (20 patients), represented by patients enrolled for orthodontic treatment in the Orthodontics and Dentofacial Orthopedics Clinic of the Medical Dental School George Emil Palade University of Medicine and Pharmacy from Targu Mures. However, because the study was undertaken at the beginning of the Covid 19 pandemic, we had to limit our exposure and thus reduce number of participants. Nevertheless, the sample size studied in this 
report has generated statistically significant results and a strong statistical power in our methodology.

\section{Conclusions}

1. Through bone remodeling biomarker analysis in the crevicular fluid, the efficiency of the orthodontic treatment can be monitored, evaluating the tissular response to the intensity of the orthodontic force.

2. The crevicular fluid volume increased $24 \mathrm{~h}$ after the initiation of the orthodontic treatment as well as the level of IL1 $\beta$.

3. The GFC levels of IL1 $\beta 24 \mathrm{~h}$ after the activation of the orthodontic treatment varied between adolescents and young adults suggesting different tissue responses in the two groups. Thus, initial tooth movement in adolescents occurs more rapidly than in young adults.

Author Contributions: Conceptualization, S.C., M.P., M.O.; methodology, S.C., P.S., M.D., E.T.; validation, M.O.; investigation, S.C., M.N.E.; resources, M.O., M.N.E., P.S., M.D.; writing—original draft preparation, S.C.; writing-S.C., E.B., M.P.; visualization, S.C., E.T.; supervision, M.P., E.B.; project administration, M.P. All authors have read and agreed to the published version of the manuscript.

Funding: This research received no external funding.

Institutional Review Board Statement: The study was conducted according to the guidelines of the Declaration of Helsinki, and approved by the Ethics Committee of George Emil Palade University of Medicine and Pharmacy (897/08 May 2020).

Informed Consent Statement: Informed consent was obtained from all subjects involved in the study.

Data Availability Statement: The data presented in this study are available on request from the corresponding author. The data are not publicly available due to patients' confidentiality reasons.

Conflicts of Interest: The authors declare no conflict of interest.

\section{References}

1. Delima, A.J.; van Dyke, T.E. Origin and function of the cellular components in gingival crevice fluid. Periodontol 2000 2003, 31, 55-76. [CrossRef]

2. Khurshid, Z.; Mali, M.; Naseem, M.; Najeeb, S.; Zafar, M.S. Human gingival crevicular fluids proteomics. An overview. Dent. J. 2017, 5, 12. [CrossRef] [PubMed]

3. Vinod, K.; Davidovitchb, Z. Cellular, molecular, and tissue-level reactions to orthodontic force. AJO-DO 2006, 129, 469. [CrossRef]

4. Caires Sobral de Aguiar, M.; Perinetti, G.; Capelli, J., Jr. The gingival crevicular fluid as a source of biomarkers to enhance efficiency of orthodontic and functional treatment of growing patients. Biomed. Res. Int. 2017, 2017, 3257235. [CrossRef]

5. Taba, M.; Kinney, J.; Kim, A.S.; Giannobile, W. Diagnostic biomarkers for oral and periodontal diseases. Dent. Clin. N. Am. 2005, 49, 551-vi. [CrossRef] [PubMed]

6. Castro, C.E.; Koss, M.A.; Lopez, M.E. Biochemical markers of the periodontal ligament. Med. Oral 2003, 8, 322-328. [PubMed]

7. Alhadlaq, A.M. Biomarkers of orthodontic tooth movement in gingival crevicular fluid: A systematic review. J. Contem. Dent. Pract. 2015, 16, 578-587. [CrossRef] [PubMed]

8. $\quad$ Lang, N.K.; Lindhe, L. Clinical Periodontology and Implant Dentistry, 6th ed.; Wiley- Blackwell: Chichester, West Sussex, UK, 2015; pp. 1297-1299. ISBN 978-0-470-67248-8.

9. Milošević-Jovčić, N.; Vujačić, A.; Konić, A.; Pavlović, J.; Todorović, V.; Glibetić, M. The role of cytokines in orthodontic tooth movement. Srp. Arh. Celok. Lek. 2012, 140, 371-378. [CrossRef]

10. Preshaw, P.M. Detection and diagnosis of periodontal conditions amenable to prevention. BMC Oral Health 2015, 15, S5. [CrossRef]

11. Sharma, S.; Tandon, P.; Nagar, A.; Singh, G.C.; Singh, A.; Chugh, V.K. A comparison of shear bond strength of orthodontic brackets bonded with four different orthodontic adhesives. J. Orthod. Sci. 2014, 3, 29-33. [CrossRef]

12. Vujacic, A.; Ristic, A.K.; Pavlovic, J.; Todorovic, V. Differences in IL-1b and IL-6 levels in the gingival crevicular fluid during acute phase of orthodontic tooth movement between juveniles and young adults. Vojn. Pregl. 2016, 74, 178. [CrossRef]

13. Teixeira, C.; Khoo, E.; Tran, J.; Chartres, I.; Liu, Y.; Thant, L.M.; Khabensky, I.; Gart, L.P.; Cisneros, G.M.; Alikhani, M. Cytokine expression and accelerated tooth movement. J. Dent. Res. 2010, 89, 1135-1141. [CrossRef]

14. Jayaprakash, P.K.; Basavanna, J.M.; Grewal, H.; Modi, P.; Sapawat, P.; Bohara, P.D. Elevated levels of Interleukin (IL)-1 $\beta$, IL6 , tumor necrosis factor- $\alpha$, epidermal growth factor, and $\beta 2$-microglobulin levels in gingival crevicular fluid during human Orthodontic tooth movement (OTM). J. Family Med. Prim. Care 2019, 8, 1602-1606. [CrossRef] 
15. Gujar, A.N.; Baeshen, H.A.; Alhazmi, A.; Bhandi, S.; Raj, A.T.; Patil, S.; Birkhed, D. Cytokine levels in gingival crevicular fluid during orthodontic treatment with aligners compared to conventional labial fixed appliances: A 3-week clinical study. Acta Odontol. Scand. 2019, 77, 471-481. [CrossRef]

16. Nunes, L.; Quintanilha, L.; Perinetti, G.; Capelli, J., Jr. Effect of orthodontic force on expression levels of ten cytokines in gingival crevicular fluid. Arch. Oral Biol. 2017, 76, 70-75. [CrossRef]

17. Zhang, Q.; Chen, B.; Zhu, D.; Yan, F. Biomarkers levels in gingival crevicular fluid of subjects with different periodontal conditions: Across-sectional study. Arch. Oral Biol. 2016, 72, 92-98. [CrossRef]

18. Mohammed, A.; Saidath, K.; Mohindroo, A.; Shetty, A.; Shama, R.; Shetty, P. Assessment and measurement of interleukin 6 in periodontal ligament tissues during orthodontic tooth movement. World J. Dent. 2019, 10, 88-92. [CrossRef]

19. Ren, Y.; Hazemeijer, H.; de Haan, B.; Qu, N.; de Vos, P. Cytokine profiles in crevicular fluid during orthodontic tooth movement of short and long durations. J. Periodontol. 2007, 78, 453-458. [CrossRef]

20. Sari, E.; Uçar, C. Interleukin 1 levels around microscrew implants during orthodontic tooth movement. Angle Orthod. 2007, 77, 1073-1078. [CrossRef]

21. Jelihovschi, I.; Drochioi, C.; Bădescu, A.; Lupusoru, R.; Munteanu, A.; Baranov, N.; Manuc, D.; Serban, I.R.; Cobzaru, R.; Rîpă, C.; et al. Comparison of sampling techniques for qPCR quantification of periodontal pathogens. Rev. Chim. 2017, 68, 2853-2856. [CrossRef]

22. Drummond, S.; Canavarro, C.; Perinetti, G.; Teles, R.; Capelli, J., Jr. The monitoring of gingival crevicular fluid volume during orthodontic treatment: A longitudinal randomized split-mouth study. Eur. J. Orthod. 2011, 34, 109-113. [CrossRef] [PubMed]

23. Vaida, L.; Mutiu, G.; Tara, I.G.; Bodog, F. An algorithm of ethical approach to the orthodontic patients. Iran. J. Public Health 2015, 44, 1296-1298. [PubMed]

24. Grzibovskis, M.; Urtane, I.; Pilmane, M. Specific signaling molecule expression in periodontal ligaments in different age groups: Pilot study. Stomatologija 2011, 13, 117-222. [PubMed]

25. Schubert, A.; Jäger, F.; Maltha, J.C.; Bartzela, T.N. Age effect on orthodontic tooth movement rate and the composition of gingival crevicular fluid: A literature review. J. Orofac. Orthop. 2020, 81, 113-125. [CrossRef] [PubMed]

26. Sfondrini, M.F.; Debiaggi, M.; Zara, F.; Brerra, R.; Comelli, M.; Bianchi, M.; Pollone, S.R.; Scribante, A. Influence of lingual bracket position on microbial and periodontal parameters in vivo. J. Appl. Oral Sci. 2012, 20,357-361. [CrossRef]

27. Kim, K.; Jung, W.S.; Cho, S.; Ahn, S.J. Changes in salivary periodontal pathogens after orthodontic treatment: An in vivo prospective study. Angle Orthod. 2016, 86, 998-1003. [CrossRef] 